Published in final edited form as:

Immunol Rev. 2006 October ; 213: 36-47.

\title{
Stranger in a strange land
}

\author{
Joan S. Hunt \\ Department of Anatomy and Cell Biology, University of Kansas Medical Center, Kansas City, KS, \\ USA.
}

\section{Summary}

Mammalian mothers and their embryos/fetuses are almost invariably genetically different, which raises the question of how the mother's immune system is diverted so as to permit cohabitation with the 'foreign' body. Several decades of research have shown that multiple cooperative systems sanction uteroplacental immune privilege. These systems include production of several varieties of soluble immunosuppressive molecules in the uterus and the placenta and strict regulation of the molecules expressed on or by placental trophoblast cells. Trophoblast, a unique lineage without counterpart in adult tissues, is in direct contact with maternal blood and tissue. The major graft rejection-promoting molecules, human leukocyte antigens (HLAs), are tightly regulated in these cells, with none of HLA-A, HLA-B, or HLA class II antigens expressed. The HLA class Ib antigens, HLA-E, HLA-F, and HLA-G, are detectable on some subpopulations. Our studies have focused on the expression, regulation, and functions of the soluble isoforms of HLA-G, which circulate in maternal blood and are present at high levels in the pregnant uterus. These isoforms are derived from the single $H L A-G$ gene by alternative splicing and are now known to have immunosuppressive properties. Ours and other studies indicate that soluble HLA-G proteins may comprise a unique tolerogenic system for establishing local immune privilege during pregnancy.

\section{Keywords}

immune privilege; pregnancy; trophoblast; HLA-G

\section{Introduction}

Despite genetic dissimilarities that should lead to immunological rejection, human fetuses are usually carried to term and healthy babies are born with satisfying regularity. Yet, during gestation, the embryo/fetus is indeed a 'stranger in a strange land', a circumstance described in a landmark science fiction work by Heinlein (1). Heinlein described the sense of both foreignness and familiarity experienced by Valentine Michael Smith, a human born on Mars who returned to Earth. Thus, the parallel: to survive, the human fetus must also adapt to a potentially hostile but simultaneously nourishing environment during maturation and development in the mother's uterus.

In the paragraphs below, the elements of the human maternal-fetal interface are described and adaptations that permit semiallogenic pregnancy in women are enumerated briefly. In particular, we discuss evidence that placental expression of the human leukocyte antigen (HLA) class Ib antigen, HLA-G, has a major impact on immune privilege in human pregnancy.

Correspondence to: Joan S. Hunt, PhD, DSc, Department of Anatomy and Cell Biology, University of Kansas Medical Center, 3901 Rainbow Boulevard, Kansas City, KS 66160, USA, Tel.: 913588 7270, Fax: 913588 7180, E-mail: jhunt@kumc.edu. 


\section{The intimate juxtaposition of maternal and fetal tissues}

Fertilization of the ovum occurs in the fallopian tube. Over the course of the next 4 days, the developing embryo moves toward the uterus, which is in the process of remodeling for implantation. The embryo is ready to implant at the blastocyst stage, the first stage at which differentiation into trophectoderm and inner cell mass cells is readily detectable. The trophectoderm generates the outermost trophoblast cells that form the placenta and the chorion membrane, whereas the inner cell mass generates the fetus, the amnion membrane, and the mesenchymal cells lying immediately beneath the trophoblast cells.

At the time of implantation, cells comprising the uterine lining (endometrium) prepare for pregnancy, a process called decidualization. In women, the uterine spiral arteries are constricted, and the embryo implants in a hypoxic environment (2). It is not until approximately week 10 of human gestation that the spiral arteries open to flood the placenta with maternal blood containing nutrients and oxygen. The decidua contains not only cells that provide nourishment to the fetus but also a carefully selected set of maternal leukocytes that are ultimately encountered by migrating, invasive fetal cells (3).

A graphic illustration of the intimate physical relationship existing between maternal and fetal tissues in human pregnancies is shown in Fig. 1. On the fetal side of the maternal-fetal interface, trophoblast cells derived from the outermost trophectoderm layer of the blastocyst form the external cell layers of the placenta and comprise the chorion membrane. These cells surround and enclose the fetus throughout pregnancy and interact directly with elements of the maternal immune system. Trophoblast cells are subdivided by anatomic location, type of differentiation, and role in inducing tolerance in the pregnant uterus. The placental villi house two subpopulations: villous cytotrophoblast (vCTB) cells and syncytialized trophoblast (sTB). The $\mathrm{vCTB}$ is a precursor cell that merges with the syncytium as the growing fetus requires an expanded system of placental support. The sTB is a single-cell layer that produces critical pregnancy hormones, such as progesterone, and is responsible for bidirectional transport of nutrients from the mother to the fetus and removal of fetal waste. Within the villi, mesenchymal cells that include early macrophages, called Hofbauer cells, fibroblasts, and undifferentiated cells that ultimately become sources of endothelial cells for the developing placental vasculature are generated from the inner cell mass of the embryo. The mesenchymal cells are contiguous with the stroma of the umbilical cord.

A third subpopulation of trophoblast cells termed the extravillous cytotrophoblast (xvCTB) cells invades the decidua (upper right panel of Fig. 1). These cells are generated from vCTB cells that proliferate, form columns, and head toward the decidualizing endometrial lining of the uterus. The point of attachment is termed the trophoblastic shell, and migrating xvCTB cells radiate from this attachment point. These cells travel with apparent ease through the interstitium to locate and invade the maternal spiral arteries, where they replace the endothelial cells. During this process, the smooth muscle cells surrounding the spiral arteries are lost. Thus, controls on vasodilation and vasoconstriction are removed, permitting maternal blood to flow over the placenta. As pregnancy progresses, these xvCTB cells regress to form the chorion membrane, which remains in immediate proximity to the maternal decidua (lower right panel of Fig. 1).

This type of placentation, where neither endothelial cells nor basement membrane intervenes between maternal blood or tissue containing leukocytes and fetal trophoblast cells, is called 'hemochorial'. The functional result is that placental and extraplacental trophoblast cells bear the major responsibility for achieving an immunologically compatible relationship between the mother and the fetus. Many of the same maternal-fetal physical arrangements of placentation are found in mice, which are popular models for studying human pregnancy (4). 


\section{Mothers and their embryos/fetuses cooperate to develop uteroplacental immune privilege}

One circumstance of immune privilege in human pregnancy is clear; the embryo/fetus survives as a consequence of cooperative interactions between the fetus and the mother [reviewed by Hunt et al. $(5,6)$ and Hunt $(7)]$.

\section{Maternal contributions}

The earliest events of tolerance generation appear to rely mainly on maternal factors. Progesterone, an immunosuppressive hormone, is produced in the ovary in the secretory phase of the cycle and in the early stages of pregnancy. Implantation is characterized by inflammatory cytokines from a wave of leukocytes, with neutrophils and macrophages predominating, as documented in the mouse (8). Thus, the mother is alerted to the implant.

Subsequently, dramatic accommodations are made in the cellular composition of the decidualizing human endometrium. As with other mucosal sites, the cycling uterus is seeded with $\mathrm{T}$ and $\mathrm{B}$ lymphocytes and with macrophages, dendritic cells, and natural killer (NK) cells. Following implantation, the pregnant uterus is redesigned as a site of innate immunity rather than acquired immunity. In women, uterine NK (uNK) cells with an unusual spectrum of markers (CD56 ${ }^{\text {bright }} \mathrm{CD} 16^{-}$) comprise approximately $20-40 \%$ of the leukocytes during the first two trimesters (until approximately week 24) and then disappear (9). In mice, these cells are readily identified until parturition, with degranulation a major feature of late gestation (10), but this is not visible in women. Macrophages are consistently present throughout gestation, constituting $10-20 \%$ of the leukocytes. $\mathrm{CD}^{+} \mathrm{CD} 25^{+}$regulatory $\mathrm{T}$ cells (Tregs) (11) are also detectable, as are small numbers of dendritic cells and $\mathrm{CD} 8^{+}$lymphocytes.

The cells of the innate immune system present in the decidua provide a measure of protection to the host (mother) against pathogens but in the absence of acquired immunity, defenses are weakened, and infections are comparatively common in the amniochorion membranes surrounding the fetus.

\section{Fetal contributions}

Trophoblast cells in the placenta and chorion membrane carry the major responsibility of driving remodeling of the endometrium and instituting tolerance to the fetal semiallograft (Fig. 2). First, trophoblast cells produce chemokines such as CXCL12/stromal cell factor-1 (12), which are capable of attracting cells of the innate immune system and may therefore play a major part in the switch from acquired to innate immunity occurring in the uterus.

Second, trophoblast cells produce general soluble immunosuppressants, such as progesterone and prostaglandins, and specific suppressor molecules. These molecules include the soluble isoforms of HLA-G and HLA-E, which are present at high levels at the maternal-fetal interface, and tolerogenic molecules, such as transforming growth factor- $\beta 1$ (TGF- $\beta 1$ ) and interleukin- 10 (IL-10). Uterine leukocytes are highly receptive to these powerful trophoblast-programming molecules. For example, uNK cells express inhibitory receptors [leukocyte immunoglobulinlike receptors (LILR)B1)] that may bind HLA-G and CD94/NKG receptors that bind HLA-E. Macrophages also express LILRB, probably mainly LILRB2, which preferentially recognize HLA-G. Dendritic cells may also be receptive to this molecule. $\mathrm{CD} 8^{+} \mathrm{T}$ cells are known to receive signals from HLA-G $(13,14)$, but the receptors are not clearly documented for this lymphocyte population in the uterus. The consequences of decidual leukocyte binding of HLA class Ib membrane and soluble antigens are discussed below. $\mathrm{CD} 4^{+} \mathrm{CD} 25^{+}$Tregs, a subpopulation of recent interest (11), may be generated through exposure to TGF- $\beta 1$, which is secreted by migrating $\mathrm{xVCTB}$ cells (15) and HLA-G-exposed macrophages (16). The role 
of IL-10 was first illuminated in the mouse by Wegmann et al. (17), who, in a burst of prescience, questioned whether pregnancy should not be considered a T-helper 2-predominant condition.

\section{Multiple tolerogenic systems protect human pregnancy}

Survival mechanisms have evolved over the course of evolution to permit the semiallogeneic embryo/fetus to reside safely within the mother, where it is protected against dangerous environmental insults. These mechanisms are both diverse and overlapping. As with other aspects of immunity, the most sophisticated mechanisms are found in humans, although those in primates are often very close, and many parallels can be found between pregnancy in humans and in rodents [reviewed by Hunt et al. and Hunt $(5,6,7)]$.

Table 1 lists specific factors for which evidence of a critical role in protecting the embryo/fetus from maternal immunity in pregnancy has accumulated. It is noted that all are features of trophoblast, whether it be soluble suppressor molecules, membrane-bound suppressor molecules, or other systems. Of these known molecules, experiments in transgenic and knockout mice have verified the importance of the complement regulatory proteins (18) but not indoleamine 2,3-dioxygenase (19). Yet, in the IDO-deficient mouse, the overlap and redundancy of protective mechanisms in pregnancy might account for lack of a phenotype, as turned out to be the case for HLA-G1 in women. When a defect in the genetic structure prevents synthesis of HLA-G1/G5, pregnancies may still survive (20), presumably because other isoforms with similar features are present. Many investigators consider lack of individuals with certain natural 'knockout' phenotypes to confirm the central role played by the gene product. A complete absence of a human population lacking all the complement regulatory proteins and all the HLA-G protein isoforms are good examples.

Mechanisms that operate in humans are often not found or are different in experimental models. For example, it has been difficult to locate an HLA-G correlate in rodents, although there is a candidate with high expression in mouse placentas and somewhat similar features (21). The primate $H L A-G$ homologue is silenced but is replaced with another similar gene. In baboons, this gene, Paan-AG, does not yield all the alternative messages that arise from $H L A-G$, and the regulatory regions are more similar to $H L A-A$ than to $H L A-G(22,23)$.

Thus, strategies developed to protect the stranger and ensure human pregnancy are of a higher degree of complexity and redundancy than in other species. Although experimental animal models are extremely helpful, the most valuable data are obtained in experiments on human tissues. In our laboratory, we first identify a potential mechanism in the human decidua/ placenta, work to establish an experimental model, and then return to human tissues to define similarities/differences from model system outcomes. The final goal is to improve fertility by understanding where, when, and what type of intervention might be helpful.

Emerging evidence supports the idea that the functions of the so-called immunological molecules may differ from their classical roles when operating at the maternal-fetal interface. One example is the presence of tumor necrosis factor (TNF) superfamily ligands in human placentas (24). Among the expressed ligands are TNF $\alpha$, TNF-related apoptosis-inducing ligand (TRAIL), and LIGHT [homologous to lymphotoxins, inducible expression, competes with herpes simplex virus (HSV) glycoprotein D for herpes virus entry mediator (HVEM), a receptor expressed on T lymphocytes], which may be at least as important to placental development and function as to immunity (25, 26, R. M. Gill, University of California and J. S. Hunt, unpublished data). Fas ligand (FasL), originally believed to have a major role in protecting the mouse (27) and the human (28) trophoblast from immune cell killing, may function, instead or in addition, to facilitate invasion and remodeling of the decidua and its vessels by $\mathrm{xvCTB}$ cells. 


\section{The HLA genes and their proteins}

The telomeric end of chromosome 6p21 includes the genes encoding HLA antigens. Many class I genes are present, but few are transcribed or translated. Most are gene fragments or pseudogenes. The expressed class I genes include class Ia (HLA-A, $H L A-B$, and $H L A-C)$ and class Ib (HLA-E, HLA-F, and $H L A-G)$. For a discussion of the different features of class Ia and class Ib genes and their antigens, see recent reviews (5-7). Of particular importance to the condition of semi-allogeneic pregnancy, the former are highly polymorphic, with many alleles, and the latter have few variants. Further, class Ia antigens are ubiquitously expressed, whereas expression of class Ib antigens may be tissue/organ specific and/or conditional. Class Ia antigens are membrane bound, whereas at least one member of the class Ib group, HLA-G, is alternatively spliced and produces transcripts that encode both membrane and soluble antigens.

Remarkably, human placental trophoblast cells express only one class Ia molecule, HLA-C, but display all three class Ib molecules. Functions of the trophoblast HLA-C antigens are as yet poorly understood but may have some indirect influence on fertility (29). The HLA class Ib antigens expressed by trophoblast cells, HLA-E, HLA-F, and HLA-G, are distinguished by low numbers of alleles that differ at the protein level. HLA-E has two alleles (30) and HLAG has five [reviewed by Ober and Aldrich (31)], but there are no allelic variants of HLA-F in the published literature. Two non-synonymous (amino acid) substitutions reported in online databases (http://genome.ucsc.edu) are likely to be uncommon in the general population. Most of the polymorphisms in the HLA-G gene do not alter the amino acid sequence; the few that do are not predicted to change secondary structures of the heavy chains (31).

\section{Structural features of HLA-G}

HLA-G was the first trophoblast HLA class I antigen to be identified and remains an antigen of great interest and focus of experimental evaluation [reviewed by Hunt et al. $(5,6)$, Hunt (7), Hunt and Orr (32), and Le Bouteiller and Mallet (33)]. For a complete discussion of structural features of HLA-G, see a recent review (5). Features important to function include the following: low numbers of functionally different alleles; generation of seven alternatively spliced transcripts, of which four are predicted to encode membrane-bound and three predicted to encode soluble proteins (Fig. 3); and generation of proteins with a truncated cytoplasmic tail, revealing a cryptic retrieval motif that may interfere with presentation of exogenous peptides. Regarding allelic polymorphism, $H L A-G$ has relatively little in its coding region (5). Nine polymorphisms have been identified to date in the exons encoding the extracellular domain, and one has been identified in the $3^{\prime}$ untranslated region (UTR). Five result in amino acid differences and four do not. One major deletion has been identified: a single base pair (bp) deletion at nucleotide 1597, which causes a frameshift at amino acid 130 (34). This deletion of a cytosine residue at codon 130 results in a null allele (called $\mathrm{G}^{*} 0105 \mathrm{~N}$ ), which does not encode functional HLA-G1 or HLA-G5 protein isoforms (20). The mutation, called $1597 \Delta \mathrm{C}$, occurs in the homozygous form in apparently healthy individuals, indicating that the HLA-G1 and HLA-G5 isoforms are not essential for fetal survival $(20,34)$. In these individuals, other isoforms presumably suffice $(20,35)$, but nonetheless, this null allele is associated with increased risk for recurrent miscarriage (36,37). Thus, HLA-G1 and/or HLA-G5 proteins are very likely to play an important role in the maintenance of pregnancy, and reduced levels of one or both may be a risk factor for recurrent miscarriage.

In the UTR, a 14-bp insertion/deletion polymorphism is present in exon 8 that may have different functional implications. When studying messenger RNA (mRNA) from term trophoblast cells, Hviid et al. (38) showed that heterozygotes for the $G^{*} 01012$ allele had reduced levels of transcripts encoding the membrane-bound isoforms (HLA-G1, HLA-G2, and HLA-G3) with the 14-bp insertion, whereas heterozygotes for the $\mathrm{G}^{*} 01013$ allele had higher levels of the HLA-G2 and/or HLA-G4 transcripts compared with the $G^{*} 01011$ allele. Although 
the significance of these findings is not clear, these differences might contribute to diseases of pregnancy, since reduced expression of HLA-G mRNA or protein in term placentas is a feature of preeclampsia (39-42) where XvCTB cell migration and infiltration of the decidua are impaired. Both the relative abundance of transcripts and the non-coding polymorphisms in $H L A-G$ have been associated with preeclampsia (43), suggesting that the regulation of expression of $H L A-G$ is influenced by genetic variation. Other associations and discussions of the impact of single bp polymorphisms have been published $[(31,44-52)$, reviewed by Hunt et al. $(5,6)$ and Hunt $(7)]$.

As for alternative splicing and the consequent isoforms (Fig. 3), the full-length isoform, HLAG1, is structurally similar to other class I genes, except for the truncated cytoplasmic tail. The $\mathrm{G} 2$ isoform results from the removal of exon 3; the resulting heavy chain cannot form heterodimers with $\beta 2$ microglobulin $\left(\beta_{2} \mathrm{~m}\right)$ and homodimerizes to form an HLA class-II-like structure $(14,53)$. HLA-G1 and HLA-G2 are also expressed as soluble proteins (called HLAG5 and HLA-G6, respectively) because of the inclusion of intron 4 sequences in the mature mRNA. HLA-G5 and HLA-G6 secreted proteins include a unique sequence of 21 amino acids that has been used to generate antibodies that specifically identify the secreted isoforms. HLAG5 may or may not associate with $\beta_{2} \mathrm{~m}$, whereas HLA-G6 does not. HLA-G3 results from the removal of exons 3 and 4 . Messages are present in placentas, but antibodies have not as yet been reported that specifically identify the protein. HLA-G4 and HLA-G7 mRNAs are scarce in placentas, and the functions of their putative protein products remain unknown.

\section{Patterns of expression of HLA-G}

The subpopulations of trophoblast that arise from progenitor cells within the trophectoderm layer of the blastocyst are readily recognized by morphology and anatomic location, although the exact differentiation pathways are not entirely clear. Investigations of rodent trophoblast cell lines have revealed some unexpected branching $(4,54)$, so some may be anticipated in human trophoblast as experimentation continues.

Our understanding of the HLA-G products of human trophoblast cells has improved with advances in technology. Previous work indicated that HLA class I antigen expression was restricted to the $\mathrm{xvCTB}$ cell population, with proteins being particularly prominent in cells immediately adjacent to the decidua in both early- and late-gestation placentas [reviewed by Hunt et al. and Hunt $(5,6,7)]$. Yet, this restriction may not be the case, as soluble isoforms have been independently reported in vCTB cells and in placental explants by several experienced teams of investigators $(55,56)$. The reason that only xvCTB cells were first thought to be the single site of synthesis of HLA-G was that the monoclonal antibody used most frequently to identify these antigens in tissue sections or isolated cells, W6/32, prefers a light chain $\left(\beta_{2} \mathrm{~m}\right) /$ heavy chain association for binding, so neither free heavy chains nor isoforms such as HLAG2 and HLA-G6 that cannot associate with $\beta_{2} \mathrm{~m}$ are readily detected. Reliable new monoclonal antibodies that are isoform specific include anti-HLA-G1 (o1G), anti-HLA-G5 (1-2C3), and HLA-G2/G6 (26-2H11) heavy chains (14,57). These show that HLA-G1 and HLA-G2/G6 are present only at the leading edge of trophoblast columns and the chorion membrane cytotrophoblast cells immediately adjacent to the decidua, whereas HLA-G5 is ubiquitous in trophoblast subpopulations.

\section{Patterns of expression and structure of HLA-G5}

In contrast to its membrane counterpart, HLA-G1, HLA-G5 mRNA and protein are identified in vCTB cells, sTB, maternal blood, and xvCTB cells (14). In vCTB cells, HLA-G5 is likely to be mainly $\beta_{2} \mathrm{~m}$-free heavy chain (P. Morales and J. S. Hunt, unpublished data), as $\beta_{2} \mathrm{~m}$ protein is missing in the vCTB cell layer and is difficult to identify in sTB. Although lacking $\beta_{2} \mathrm{~m}$ protein, $\beta_{2} \mathrm{~m}$ mRNA is present in both subpopulations, which yields another example of 
untranslated messages in this organ that comprise an important reservoir of human transcripts for cloning. Preliminary experiments suggest that the HLA-G5 produced in VCTB cells consists of disulfide-bonded heavy chain dimers and multimers (P. Morales and J. S. Hunt), which interact differently with HLA-G receptors than heavy chain: light chain heterodimers (58).

\section{Patterns of expression and structure of HLA-G6}

The monoclonal antibody recognizing HLA-G2 and HLA-G6 shows that one or another or both of these isoforms are prominent in/on XVCTB cells distal to the placental villi, XvCTB cells infiltrating the decidua, and some chorion membrane $\mathrm{xvCTB}$ cells (14). Interestingly, HLA-G2/G6 is located in many of the same cells as HLA-G1, suggesting that in women carrying the null allele $\left(\mathrm{G}^{*} 0105 \mathrm{~N}\right)$ that does not encode functional HLA-G1 or HLA-G5 protein isoforms yet have uneventful pregnancies, HLA-G2/G6, which in the absence of the $\alpha 2$ domain form only heavy chain homodimers (Fig. 3), may comprise an adequate substitute (20). Fig. 4 illustrates the sequence of HLA-G isoform development that we believe may occur in trophoblast columns as they near the decidua.

\section{Clinical implications}

The soluble isoforms, mainly HLA-G5, circulate in mothers' blood throughout pregnancy (59, J. L. Pace and J. S. Hunt, University of California, unpublished data). Furthermore, soluble HLA-G is produced by some but not by all preimplantation embryos (60). In some of these experiments, which remain controversial, high levels of soluble HLA-G in the supernatant culture media of embryos cultured in vitro indicate an ability to implant successfully. For a discussion of the usefulness of this observation, see recent reviews (5-7). Because of links between low abundance of HLA-G proteins and the possibility of hindrance to optimum pregnancy, described above, clinicians are now looking for enzyme-linked immunosorbent assays that will be useful in identifying patients with suboptimal fertility. Our work suggests that these assays would most profitably focus on the soluble isoform HLA-G5 and use reagents that would identify both $\beta_{2} \mathrm{~m}$-associated and non-associated heavy chains of HLA-G5.

\section{Receptors for HLA-G}

Due to an emerging understanding of inhibitory receptors on immune cells that block activating signals, the original view that the HLA class I antigens on trophoblast cells might be promoting self-destruction has required revision. It remains clear that because of their low polymorphism, the class Ib antigens are preferable to class Ia when considering the potential of trophoblast antigens to stimulate destructive pathways. However, experiments in our laboratory have shown that even when maternal and paternal alleles of HLA-G differ, antibodies synthesized in mothers do not place restrictions on fertility or successful pregnancy (61). These mothers have multiple normal pregnancies, as is also the case for mothers synthesizing anti-paternal HLA-A, HLA-B, and HLA-D.

Immunologists have met the challenge of understanding how migrating xvCTB cells might use class Ib antigens. HLA-E binds to the lymphocyte-inhibitory receptor CD94/NKG2A (62). Wooden et al. (63) have recently reported that this receptor inhibits NK-cell-mediated lysis. uNK cells, the most populous leukocyte subpopulation in the decidua during the first two trimesters, could encounter trophoblast cells bearing HLA-E and would be predicted to arrive at an anti-inflammatory profile following receptor/ligand interactions. For a recent review of HLA-E and HLA-F in pregnancy, see Ishitani et al. (64). Interestingly, all three class Ib antigens have been reported as coexpressed on $\mathrm{xVCTB}$ cells by this group (57).

For HLA-G, the major receptors on leukocytes are the leukocyte-inhibitory receptors (LILRB), formerly known as the immunoglobulin-like transcript (ILT) receptors [reviewed by Allan $e t$ al. (65) and Brown et al. (66)] (Fig. 5). Binding of trophoblast cell HLA-G to these receptors 
so as to inhibit activating signals on decidual leukocytes may be the answer to the question of why trophoblast cells should need to express HLA class I (Fig. 6).

LILRBs are expressed by $\mathrm{T}$ and B lymphocytes and also by NK cells and mononuclear phagocytes $(65,66)$, and LILRBs abrogate activating signals received by these cells (Fig. 6). Although LILRB1 (ILT2) appears to be the main binding protein for lymphocytes, LILRB2 (ILT4) may be the main receptor for HLA-G, which is exhibited by monocyte/macrophages, the second most populous leukocyte population in the human decidua (3). However, LILRB1 has not been formally excluded as a mononuclear phagocyte recognition entity, and our recent experiments in a macrophage cell line suggest that these two receptors may exhibit isoformspecific binding (16). This novel finding illustrates the point that little is known of how HLAG-activated signaling pathways, which include tyrosine phosphorylation, SH2-domaincontaining phosphatase-1 association, and calcium regulation (Fig. 6), may be translated into expression of specific genes in decidual leukocytes, as required for programming the cells into pregnancy-appropriate behavior.

\section{Regulation in trophoblast cells}

Previous work in our laboratory showed that HLA-G mRNA may be modestly upregulated by interferons (IFNs), but cell surface protein expression in trophoblast tumor cells, whether positive (JEG-3 cells) or negative (Jar cells), remains unaltered (67). The Ober group (52) has described differential regulation related to specific polymorphisms in the promoter region using transient transfection of reporter constructs into the same tumor cells.

We recently initiated investigations of other regulatory molecules and circumstances normally found at the maternal-fetal interface in an effort to understand how differential expression of HLA-G5 and HLA-G6 in trophoblast cell sub-populations is achieved. Not surprisingly, low oxygen induced all HLA-G transcripts (68). This outcome was expected because expression of the proteins is high in early human gestation, where oxygen is low (2). Unexpectedly, preliminary experiments suggest that epidermal growth factor, a well-known trophoblast growth and syncytialization factor, and the substrate on which vCTB cells from term placentas are cultured, notably collagen IV, have dramatic effects. Our postulates regarding these regulatory factors/conditions are shown in Fig. 7. An immunohistochemical study of antigens in $\mathrm{VCTB}$ cells cultured on plastic or collagen-IV-coated plastic plates for $48 \mathrm{~h}$ is shown in Fig. 8. Note the increased size of the intracellular inclusions and increased intensity of staining for HLA-G5 without change in HLA-G6.

\section{Functions of HLA-G: targeting immune cells}

Many investigations into the functions of HLA-G have been conducted over the past several years and are summarized in recent reviews (5-7). These studies have yielded an overall impression of tolerance induction in immune cells by the proteins derived from the $H L A-G$ gene. Effects include impact on NK cell killing, migration, and cell viability; proliferation and IFN $\gamma$ production; regulation of cytokine production in blood mononuclear cells and cytotoxic T lymphocytes (CTLs); suppression of CTL killing and viability; inhibition of proliferation and induction of a suppressive phenotype in T-helper cells; and alteration of dendritic cell stimulatory capacity and maturation of this lineage.

Immunosuppression is clearly the direction taken by leukocytes exposed to the recombinant proteins we generated in HEK293 cells several years ago. We first showed that both recombinant HLA-G5 (rHLA-G5) and rHLA-G6 reduced CD8 mRNA and protein in IFN $\gamma$ activated blood mononuclear cells without affecting CD3 or causing cell death (14). Our results contrasted with those of Fournel et al. (13), who had reported earlier that tumor cell HLA-G killed phytohemagglutinin-activated $T$ cells through induction of the Fas/FasL pathway. We 
continue to see no killing by our recombinant proteins when used in other types of experiments. Our second group of functional experiments focused on the monocyte/macrophage lineage. Here, studies on the U937 myelomonocytic cell line, differentiated with phorbol myristate acetate and activated with IFN $\gamma$, indicated that both rHLA-G5 and rHLA-G6 induced production of TGF- $\beta 1$ in a dose-dependent manner (16). By contrast, production of a second immunosuppressive cytokine, IL-10, was inhibited at high doses of the recombinant proteins, and production of inflammation-associated cytokines such as TNF $\alpha$ was not altered. In all experiments where effects were observed, rHLA-G5 in molar equivalency with rHLA-G6 was 10 -fold more effective. Since that time, we have repeated the TGF- $\beta 1$ experiments using normal blood monocytes and achieved the same results. From these studies, it is possible to conclude that (i) the major effects of HLA-G5 and HLA-G6 will be local with a concentration gradient likely, (ii) HLA-G5 will be a more effective immunosuppressant than HLA-G6, (iii) CTLs may be less effective in recognizing HLA class-I-presented foreign peptides, and (iv) macrophages, which are steady inhabitants of the pregnant uterus, will be selectively driven to produce a specific cytokine that has not only immunosuppres-sive properties but also multiple additional functions (69).

\section{Perspectives}

HLA-G is now extensively documented as a major potential promoter of tolerance at the human maternal-fetal interface. Two primate models have been developed that may permit closing the cause and the effect loop. The monkey and the baboon, while not perfect, can certainly be used to conduct some of the inhibition experiments that are not permissible in women.

Does anything interesting or important remain to be done? We believe the answer to this is a firm 'Yes', as neither we nor others know everything that is required to take HLA-G to the marketplace. To move in that direction, our laboratory is working toward generating information on the structure(s) of the HLA-G5 produced in placentas. Ultimately, we hope to learn whether treating patients with recombinant proteins could improve fertility, as might be the case in women carrying specific low HLA-G abundance alleles. Because the structure(s) of the soluble HLA-G antigens may influence receptor binding, defining the crystal structures of HLA-G5 and HLA-G6 may be critical and may aid in predicting whether HLA-G5 would be an effective therapeutic agent. There is as yet no information on whether the homodimers produced in vCTB cells compete with membrane HLA-G1 for binding, thus acting as an inhibitor of the inhibitor, or whether they themselves bind efficiently and initiate interruption of activating signals.

A second basic science question could also affect therapies. We would like to improve our understanding of mechanisms underlying the ability of soluble HLA-G proteins to alter cytokine production in mononuclear phagocytes. Unpublished DNA micro-array experiments, where IFN $\gamma$-activated monocytes were treated with rHLA-G5 or rHLA-G6, indicate that the eukaryotic rHLA-G proteins, which are unlike fusion proteins in that they contain no lipopolysaccharide, have many effects on the synaptosome-associated protein (SNAP) and soluble N-ethylmaleimide-sensitive factor attachment protein receptor (SNARE) proteins that are involved in processing and exocytosis (R. H. McIntire and J. S. Hunt, University of California, unpublished data). How activated macrophages that encounter HLA-G5 and HLAG6 are directed into production of an immunosuppressive/multifunctional cytokine rather than inflammation-associated cytokines such as TNFa is an intriguing central question in immune cell biology that relates to protein trafficking pathways. Fig. 9 shows immunofluorescent localization of one of the SNAREs, syntaxin-4, for which we have preliminary evidence of modulation in mononuclear cells by rHLA-G5 (D. Wheaton and J. S. Hunt, unpublished data).

In summary, we expect the field of HLA-G biology, which has spawned its own highly successful biannual international meeting, to expand exponentially. This natural product is now 
recognized not only for its profound effects during pregnancy, where it appears to have a major role in welcoming the stranger, but also for its potential to impact equally successful but uninvited and unwanted instances of tolerance, such those evident in many cancers (70).

\section{Acknowledgements}

The author wishes to express her gratitude for the many excellent contributions of technical associates, graduate students, post-doctoral fellows, and visiting scientists to this work. The studies are supported by grants from the National Institutes of Health to JSH (HD24212; HD33994, Project IV; HD39878, Project III), the CONRAD Program for Contraceptive Research \& Development (CICCR), the Kansas University Biomedical Research Training Program, core facilities of the Kansas Reproductive Sciences Center (U54 HD33994, P.F. Terranova, P.I.), and the Kansas IDeA Network for Biomedical Research Excellence (P20 RR16475, J.S. Hunt, P.I.).

\section{References}

1. Heinlein, RA. New York: GP Putnam's Sons; 1961. Stranger in a Strange Land.

2. Burton GJ, Hempstock J, Jauniaux E. Oxygen, early embryonic metabolism and free radical-mediated embryopathies. Reprod Biomed Online 2002;6:84-96. [PubMed: 12626148]

3. Bulmer JN, Pace D, Ritson A. Immunoregulatory cells in human decidua: morphology, immunohistochemistry and function. Reprod Nutr Dev 1988;28:1599-1613. [PubMed: 3073448]

4. Rossant J, Cross JC. Placental development: lessons from mouse mutants. Nat Rev Genet 2001;2:538548. [PubMed: 11433360]

5. Hunt JS, Petroff MG, McIntire RH, Ober C. HLA-G and immune tolerance in pregnancy. FASEB J 2005;19:681-693. [PubMed: 15857883]

6. Hunt, JS.; McIntire, RH.; Petroff, MG. Immunobiology of human pregnancy. In: Neill, JD., editor. Knobil and Neill's Physiology of Reproduction. 3rd edn. 2. St Louis: Elsevier/ Academic Press; p. 2006p. 2759-2785.

7. Hunt, JS. Major histocompatibility antigens in reproduction. In: Glasser, S.; Alpin, J.; Guidice, L.; Tabibzadeh, S., editors. The Endometrium. London and New York: Taylor \& Francis; 2002. p. 405-415.

8. McMaster MT, Newton RC, Dey SK, Andrews GK. Activation and distribution of inflammatory cells in the mouse uterus during the preimplantation period. J Immunol 1992;148:1699-1705. [PubMed: 1541814]

9. Moffett-King A. Natural killer cells and pregnancy. Nat Rev Immunol 2002;2:656-663. [PubMed: 12209134]

10. Croy BA, et al. Uterine natural killer cells: insights into their cellular and molecular biology from mouse modeling. Reproduction 2003;126:149-160. [PubMed: 12887272]

11. Heikkinen J, Mottonen M, Alanen A, Lassila O. Phenotypic characterization of regulatory T cells in the human decidua. Clin Exp Immunol 2004;136:373-378. [PubMed: 15086404]

12. Wu X, Jin LP, Yuan MM, Zhu Y, Wang MY, Li DJ. Human first-trimester trophoblast cells recruit CD56brightCD16- NK cells into decidua by way of expressing and secreting of CXCL12/stromal cell-derived factor-1. J Immunol 2005;175:61-68. [PubMed: 15972632]

13. Fournel S, et al. Cutting edge: soluble HLA-G1 triggers CD95/CD95 ligand-mediated apoptosis in activated CD8+ cells by interacting with CD8. J Immunol 2000;164:6100-6104. [PubMed: 10843658]

14. Morales PJ, et al. Placental cell expression of HLA-G2 isoforms is limited to the invasive trophoblast phenotype. J Immunol 2003;171:6215-6224. [PubMed: 14634138]

15. Lysiak JJ, Hunt J, Pringle GA, Lala PK. Localization of transforming growth factor beta and its natural inhibitor decorin in the human placenta and decidua throughout gestation. Placenta 1995;16:221231. [PubMed: 7638106]

16. McIntire RH, Morales PJ, Petroff MG, Colonna M, Hunt JS. Recombinant HLA-G5 and -G6 drive U937 myelomonocytic cell production of TGF-\{beta\}1. J Leukoc Biol 2004;76:1220-1228.

[PubMed: 15459235] 
17. Wegmann TG, Lin H, Guilbert L, Mosmann TR. Bidirectional cytokine interactions in the maternalfetal relationship: is successful pregnancy a TH2 phenomenon? Immunol Today 1993;14:353-356. [PubMed: 8363725]

18. Xu C, Mao D, Holers VM, Palanca B, Cheng AM, Molina H. A critical role for murine complement regulatory crry in fetomaternal tolerance. Science 2000;287:498-501. [PubMed: 10642554]

19. Baban B, Chandler P, McCool D, Marshall B, Munn DH, Mellor AL. Indoleamine 2, 3-dioxygenase expression is restricted to fetal trophoblast giant cells during murine gestation and is maternal genome specific. J Reprod Immunol 2004;61:67-77. [PubMed: 15063630]

20. Ober C, et al. HLA-G1 protein expression is not essential for fetal survival. Placenta 1998;19:127132. [PubMed: 9548178]

21. Cao W, Brenner CA, Alikani M, Cohen J, Warner CM. Search for a human homologue of the mouse Ped gene. Mol Hum Reprod 1999;5:541-547. [PubMed: 10341001]

22. Langat DK, Morales PJ, Fazleabas AT, Mwenda JM, Hunt JS. The olive baboon (Papio anubis): a potential animal model to study the function of human leukocyte antigen-G (HLA-G). Gynecol Obstet Invest 2004;57:33-36. [PubMed: 14974449]

23. Langat DK, Morales PJ, Fazleabas AT, Mwenda JM, Hunt JS. Potential regulatory sequences in the untranslated regions of the baboon MHC class Ib gene, Paan-AG, more closely resemble those in the human MHC class Ia genes than those in the class Ib gene, HLA-G. Immunogenetics 2004;56:657666. [PubMed: 15578264]

24. Phillips TA, Ni J, Hunt JS. Death-inducing tumor necrosis factor (TNF) superfamily ligands and receptors are transcribed in human placentas, cytotrophoblasts, placental macrophages and placental cell lines. Placenta 2001;22:663-672. [PubMed: 11597186]

25. Hunt JS, Chen HL, Miller L. Tumor necrosis factors: pivotal factors in pregnancy? Biol Reprod 1996;54:554-562. [PubMed: 8835376]

26. Phillips TA, Ni J, Pan G, Ruben SM, Wei YF, Hunt JS. TRAIL (Apo-2L) and TRAIL receptors in human placentas: implications for tumor protection and immune privilege. J Immunol 1999;162:6053-6059. [PubMed: 10229846]

27. Hunt JS, Vassmer D, Ferguson TA, Miller L. Fas ligand is positioned in mouse uterus and placenta to prevent trafficking of activated leukocytes between the mother and the conceptus. J Immunol 1997;158:4122-4128. [PubMed: 9126971]

28. Kauma SW, Huff TF, Hayes N, Nilkaeo A. Placental Fas ligand expression is a mechanism for maternal immune tolerance to the fetus. J Clin Endocrinol Metab 1999;84:2188-2194. [PubMed: 10372730]

29. Hiby SE, et al. Combinations of maternal KIR and fetal HLA-C genes influence the risk of preeclampsia and reproductive success. J Exp Med 2004;200:957-965. [PubMed: 15477349]

30. Grimsley C, Ober C. Population genetic studies of HLA-E: evidence for selection. Hum Immunol 1997;52:33-40. [PubMed: 9021407]

31. Ober C, Aldrich CL. HLA-G polymorphisms: neutral evolution or novel function? J Reprod Immunol 1997;36:1-21. [PubMed: 9430736]

32. Hunt JS, Orr HT. HLA and maternal-fetal recognition. FASEB J 1992;6:2344-2348. [PubMed: 1544544]

33. Le Bouteiller P, Mallet V. HLA-G and pregnancy. Rev Reprod 1997;2:7-13. [PubMed: 9414460]

34. Suárez MB, et al. A new HLA-G allele (HLA-G*0105N) and its distribution in the Spanish population. Immunogenetics 1997;45:464-465. [PubMed: 9089111]

35. Menier C, Riteau B, Dausset J, Carosella ED, Rouas-Freiss N. HLA-G truncated isoforms can substitute for HLA-G1 in fetal survival. Hum Immunol 2000;61:1118-1125. [PubMed: 11137216]

36. Aldrich CL, et al. HLA-G genotypes and pregnancy outcome in couples with unexplained recurrent miscarriage. Mol Hum Reprod 2001;7:1162-1172.

37. Pfeiffer KA, Fimmers R, Engels G, van Der Ven H, van Der Ven K. The HLA-G genotype is potentially associated with idiopathic recurrent spontaneous abortion. Mol Hum Reprod 2001;7:373378. [PubMed: 11279300]

38. Hviid TV, Hylenius S, Rorbye C, Nielsen LG. HLA-G allelic variants are associated with differences in the HLA-G mRNA isoform profile and HLA-G mRNA levels. Immunogenetics 2003;55:63-79. [PubMed: 12712263] 
39. Goldman-Wohl DS, et al. Lack of human leukocyte antigen-G expression in extravillous trophoblasts is associated with preeclampsia. Mol Hum Reprod 2000;6:88-95. [PubMed: 10611266]

40. Lim KH, et al. Human cytotrophoblast differentiation/invasion is abnormal in preeclampsia. Am J Pathol 1997;151:1809-1818. [PubMed: 9403732]

41. Aldrich C, Verp MS, Walker MA, Ober C. A null mutation in HLA-G is not associated with preeclampsia or intrauterine growth retardation. J Reprod Immunol 2000;47:41-48. [PubMed: 10779589]

42. O'Brien M, et al. Altered HLA-G transcription in preeclampsia is associated with allele specific inheritance: possible role of the HLA-G gene in susceptibility to the disease. Cell Mol Life Sci 2001;58:1943-1949. [PubMed: 11766889]

43. Redman CW, Sargent IL. Latest advances in understanding preeclampsia. Science 2005;308:15921594. [PubMed: 15947178]

44. Hviid TV, et al. Characterization of a new HLA-G allele encoding a nonconservative amino acid substitution in the alpha3 domain (exon 4) and its relevance to certain complications in pregnancy. Immunogenetics 2001;53:48-53. [PubMed: 11261931]

45. Harrison GA, Humphrey KE, Jakobsen IB, Cooper DW. A 14 bp deletion polymorphism in the HLAG gene. Hum Mol Genet 1993;2:2200. [PubMed: 8111399]

46. Hiby SE, King A, Sharkey A, Loke YW. Molecular studies of trophoblast HLA-G: polymorphism, isoforms, imprinting and expression in preimplantation embryo. Tissue Antigens 1999;53:1-13. [PubMed: 10082426]

47. Rousseau P, Le Discorde M, Mouillot G, Marcou C, Carosella ED, Moreau P. The 14 bp deletioninsertion polymorphism in the 3' UT region of the HLA-G gene influences HLA-G mRNA stability. Hum Immunol 2003;64:1005-1010. [PubMed: 14602228]

48. Hviid TV, Hylenius S, Rorbye C, Nielsen LG. HLA-G allelic variants are associated with differences in the HLA-G mRNA isoform profile and HLA-G mRNA levels. Immunogenetics 2003;5:63-79. [PubMed: 12712263]

49. Aldrich C, Verp MS, Walker MA, Ober C. A null mutation in HLA-G is not associated with preeclampsia or intrauterine growth retardation. J Reprod Immunol 2000;47:41-48. [PubMed: 10779589]

50. Hviid TV, Sorensen S, Morling N. Polymorphism in the regulatory region located more than 1.1 kilobases $5^{\prime}$ to the start site of transcription, the promoter region, and exon 1 of the HLA-G gene. Hum Immunol 1999;60:1237-1244. [PubMed: 10626737]

51 . Ober C, et al. Variation in the HLA-G promoter region influences miscarriage rates. Am J Hum Genet 2003;72:1425-1435. [PubMed: 12721954]

52. Ober C, Billstrand C, Kuldanek S, Tan Z. The miscarriage-associated HLA-G -725 allele influences transcription rates in JEG-3 cells. Hum Reprod 2006;21:1743-1748. [PubMed: 16501035]

53. Ishitani A, Geraghty DE. Alternative splicing of HLA-G transcripts yields proteins with primary structures resembling both class I and class II antigens. Proc Nat Acad Sci U S A 1992;89:39473951.

54. Soares MJ. The prolactin and growth hormone families: pregnancy-specific hormones/cytokines at the maternal-fetal interface. Reprod Biol Endocrinol 2004;2:51. [PubMed: 15236651]

55. Le Bouteiller P. Human villous trophoblast and the lack of intron 4-retaining soluble HLA-G secretion: beware of possible methodological biases. Mol Hum Reprod 2005;11:711-713. [PubMed: 16330471]

56. Hunt JS, Geraghty DE. Soluble HLA-G isoforms, HLA-G5 and HLA-G6: technical deficiencies lead to misinterpretations. Mol Hum Reprod 2005;11:715-717. [PubMed: 16330470]

57. Ishitani A, et al. Protein expression and peptide binding suggest unique and interacting functional roles for HLA-E, F, and G in maternal-placental immune recognition. J Immunol 2003;171:13761384. [PubMed: 12874228]

58. Gonen-Gross T, et al. The CD84J/leukocyte inhibitory receptor-2 distinguishes between conformed and $\beta 2$-microglobulin-free HLA-G molecules. J Immunol 2005;175:4866-4874. [PubMed: $16210588]$

59. Hunt JS, Jadhav L, Chu W, Geraghty DE, Ober C. Soluble HLA-G circulates in maternal blood during pregnancy. Am J Obstet Gynecol 2000;183:682-688. [PubMed: 10992193] 
60. Yao YQ, Barlow DH, Sargent IL. Differential expression of alternatively spliced transcripts of HLA$\mathrm{G}$ in human preimplantation embryos and inner cell masses. J Immunol 2005;175:8379-8385. [PubMed: 16339579]

61. Hunt JS, Pace JL, Morales PJ, Ober C. Immunogenicity of the soluble isoforms of HLA-G. Mol Hum Reprod 2003;9:729-735. [PubMed: 14561816]

62. Lee N, et al. HLA-E is a major ligand for the natural killer inhibitory receptor CD94/ NKG2A. Proc Natl Acad Sci U S A 1998;95:5199-5204. [PubMed: 9560253]

63. Wooden SL, Kalb SR, Cotter RJ, Soloski MJ. Cutting edge: HLA-E binds a peptide derived from the ATP-binding cassette transporter multidrug resistance-associated protein 7 and inhibits NK cellmediated lysis. J Immunol 2005;175:1383-1387. [PubMed: 16034073]

64. Ishitani A, Sageshima N, Hatake K. The involvement of HLA-E and -F in pregnancy. J Reprod Immunol 2006;69:101-113. [PubMed: 16487601]

65. Allan DS, McMichael AJ, Braud VM. The ILT family of leukocyte receptors. Immunobiology 2000;202:34-41. [PubMed: 10879687]

66. Brown D, Trowsdale J, Allen R. The LILR family: modulators of innate and adaptive immune pathways in health and disease. Tissue Antigens 2004;64:215-225. [PubMed: 15304001]

67. Yang Y, Geraghty DE, Hunt JS. Cytokine regulation of HLA-G expression in human trophoblast cell lines. J Reprod Immunol 1995;29:179-195. [PubMed: 8636924]

68. Hunt JS, Langat D, McIntire RH, Morales P. The role of HLA-G in human pregnancy. Reprod Biol Endocrinol. (in press)

69. Li MO, Wan YY, Sanjabi S, Robertson AK, Flavell RA. Transforming growth factor-beta regulation of immune responses. Annu Rev Immunol 2006;24:99-146. [PubMed: 16551245]

70. Rouas-Freiss N, Moreau P, Ferrone S, Carosella ED. HLA-G proteins in cancer: do they provide tumor cells with an escape mechanism? Cancer Res 2005;65:10139-10144. [PubMed: 16287995]

71. Kemper C, Verbsky JW, Price JD, Atkinson JP. T-cell stimulation and regulation: with complements from CD46. Immunol Res 2005;32:31-44. [PubMed: 16106057]

72. Ragusa A, et al. Progesterone supplement in pregnancy: an immunologic therapy? Lupus 2004;13:639-642. [PubMed: 15485093]

73. Hunt JS, Miller L, Platt JS. Hormonal regulation of uterine macrophages. Dev Immunol 1998;6:105110. [PubMed: 9716911]

74. Slukvin II, Lunn DP, Watkins DI, Golos TG. Placental expression of the nonclassical MHC class I molecule Mamu-AG at implantation in the rhesus monkey. Proc Natl Acad Sci U S A 2000;97:91049109. [PubMed: 10922066]

75. Baban B, Chandler P, McCool D, Marshall B, Munn DH, Mellor AL. Indoleamine 2,3-dioxygenase expression is restricted to fetal trophoblast giant cells during murine gestation and is maternal genome specific. J Reprod Immunol 2004;61:67-77. [PubMed: 15063630]

76. Lien E, Liabakk NB, Austgulen R. Detection of soluble receptors for tumor necrosis factor, interleukin-2 and interleukin-6 in retroplacental serum from normal pregnant women. Gynecol Obstet Invest 1996;41:1-4. [PubMed: 8821875]

77. Petroff MG, Chen L, Phillips TA, Azzola D, Sedlmayr P, Hunt JS. B7 family molecules are favorably positioned at the human maternal-fetal interface. Biol Reprod 2003;68:1496-1504. [PubMed: 12606489] 


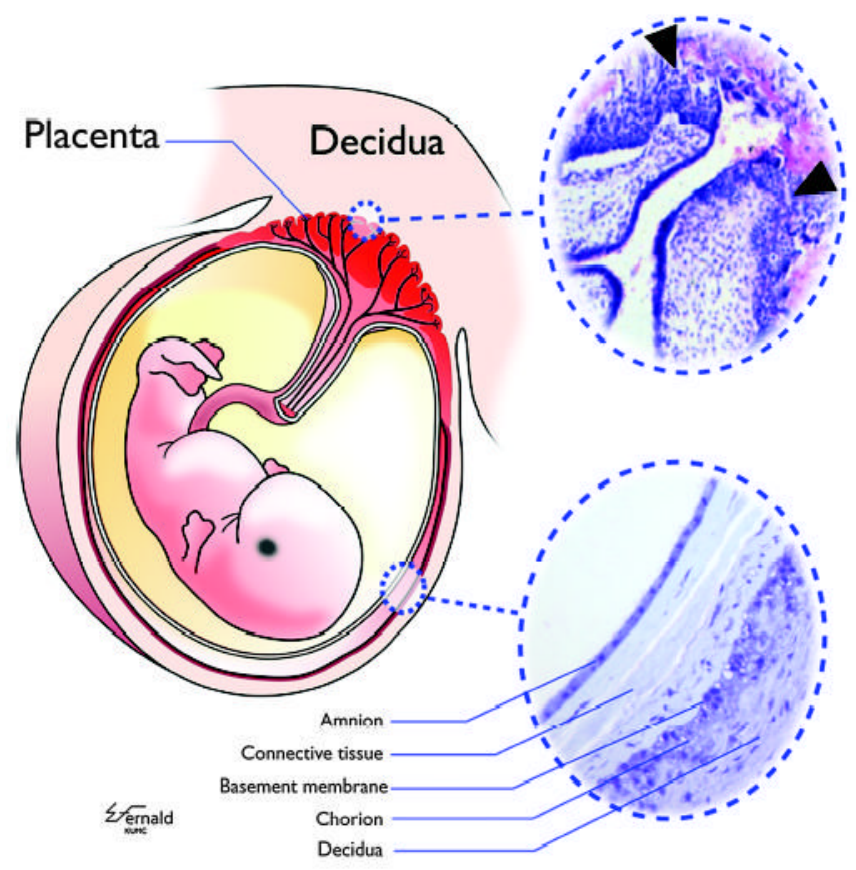

Fig. 1. A schematic drawing of the human fetus contained within the uterus

Within the uterus, the embryo/fetus is entirely surrounded and encased by a shell of trophoblast cells present in the placenta and the chorion membrane (colored red in left figure). vCTB cells in placental villi serve as the progenitors for all differentiated trophoblast cell subpopulations, including the sTB layer, which is exposed continuously to maternal blood, and the xvCTB cells that migrate into the modified uterine endometrium, which is termed the decidua. (Upper right insert) CTB cells proliferate and migrate into the decidua (arrowheads), attaching the placental villi to the mother and facilitating certain critical physiological events required for successful pregnancy. (Lower right insert) The amnion membrane, comprising a single layer of epithelial cells, is a strong sac holding the fetus suspended in amniotic fluid. The amnion is separated from the chorion membrane CTB cells, which are derived from the migrating xvCTB cells, by connective tissue. The chorion CTB cells are directly apposed to maternal decidual cells. The inserts are photomicrographs of hematoxylin and eosin-stained placenta of 5 weeks of gestation (upper right) and an amniochorion (lower right) at termination of pregnancy. Modified from Hunt et al.(5). 


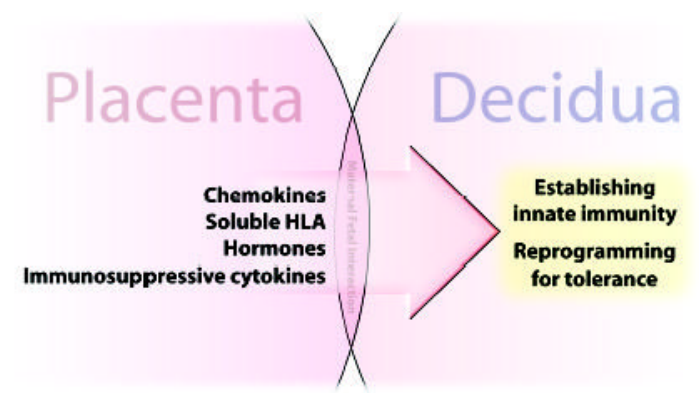

Fig. 2. Soluble products of the fetal placenta drive tolerance in the pregnant uterus The fetal placenta is directly apposed and is in contact with the decidua throughout pregnancy. Placental cells, mainly vCTB and sTB within the villi, synthesize soluble substances that program maternal immune cells in the adjacent decidua. Not shown in this figure are the substances on trophoblast cell membranes that have complementary functions. 


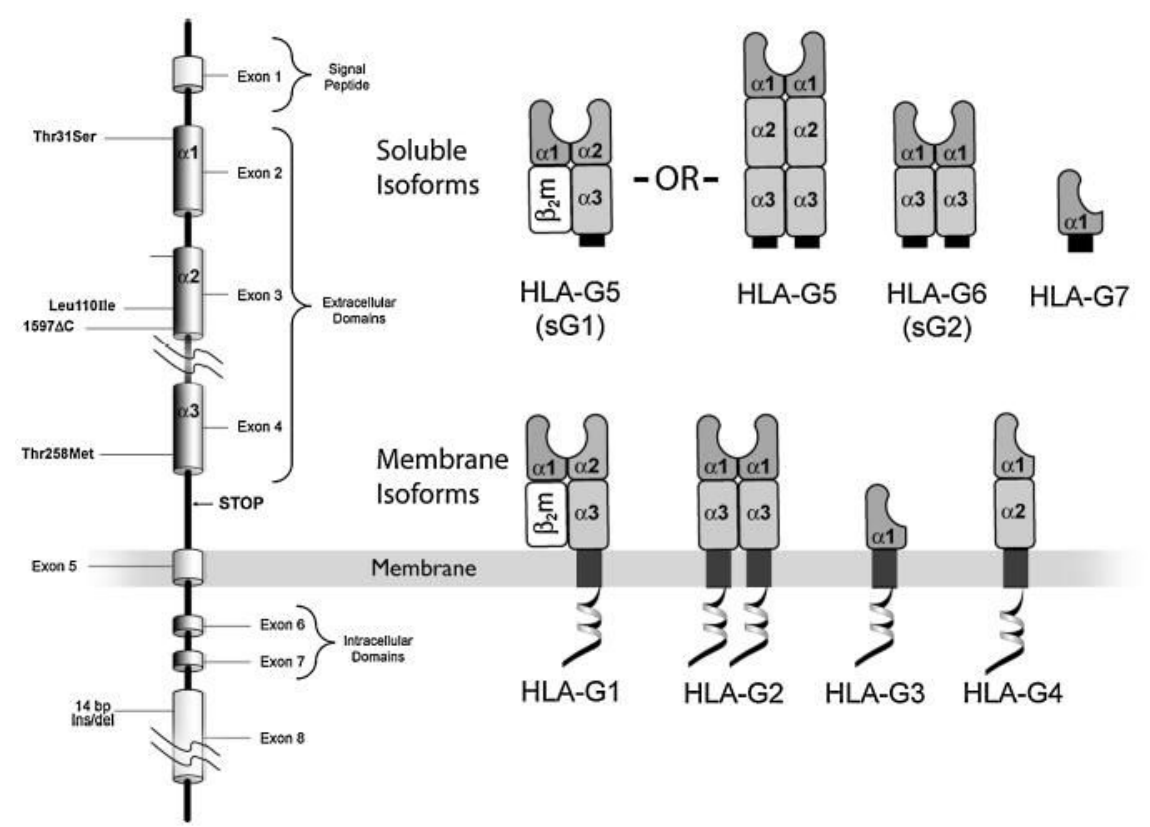

Fig. 3. Multiple HLA-G proteins result from alternative splicing of the single HLA-G mRNA (Left) The eight exons of the HLA-G gene are arranged in the same sequence as other HLA class I genes. Unlike the other HLA class I genes, the $H L A-G$ gene is alternatively spliced to yield seven transcripts. (Right) Four messages encode membrane isoforms (HLA-G1, HLAG2, HLA-G3, and HLA-G4), and two encode soluble isoforms (HLA-G5 and HLA-G6, also known as sG1 and sG2, respectively). A stop sequence in intron 4 results in HLA-G5 and HLAG6, and a stop codon in intron 2 results in a third, HLA-G7. HLA-G1 and HLA-G5 produced in some but not in all types of cells associate with light chain, $\beta_{2} \mathrm{~m}$, whereas other isoforms do not. 


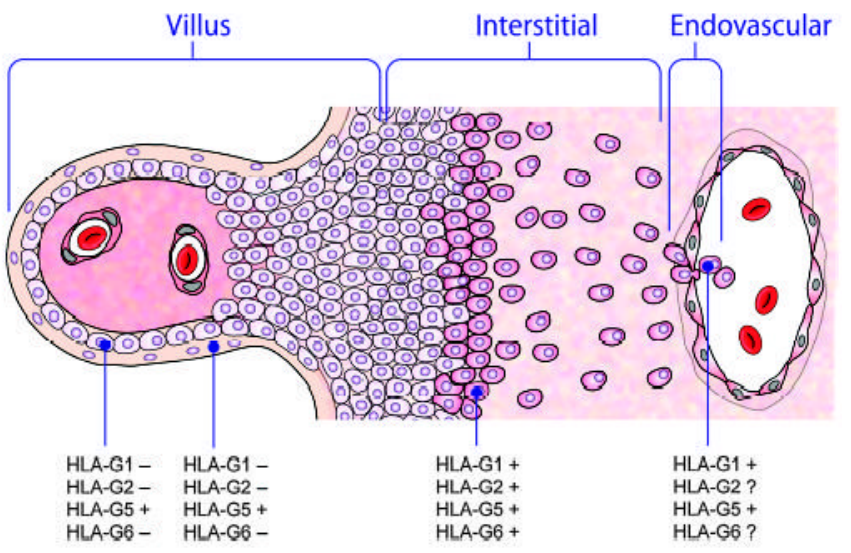

Fig. 4. HLA-G protein expression related to column formation and invasion of the decidua HLA-G5, a soluble glycoprotein, is the only isoform that is expressed in all subpopulations of trophoblast cells, including those in the villous (left), those in the column, and those forming the trophoblastic shell that attaches to the decidua. All tested isoforms appear to be expressed in the trophoblastic shell and to be maintained as the cells progress through the interstitum of the decidua and invade the spiral arteries. 


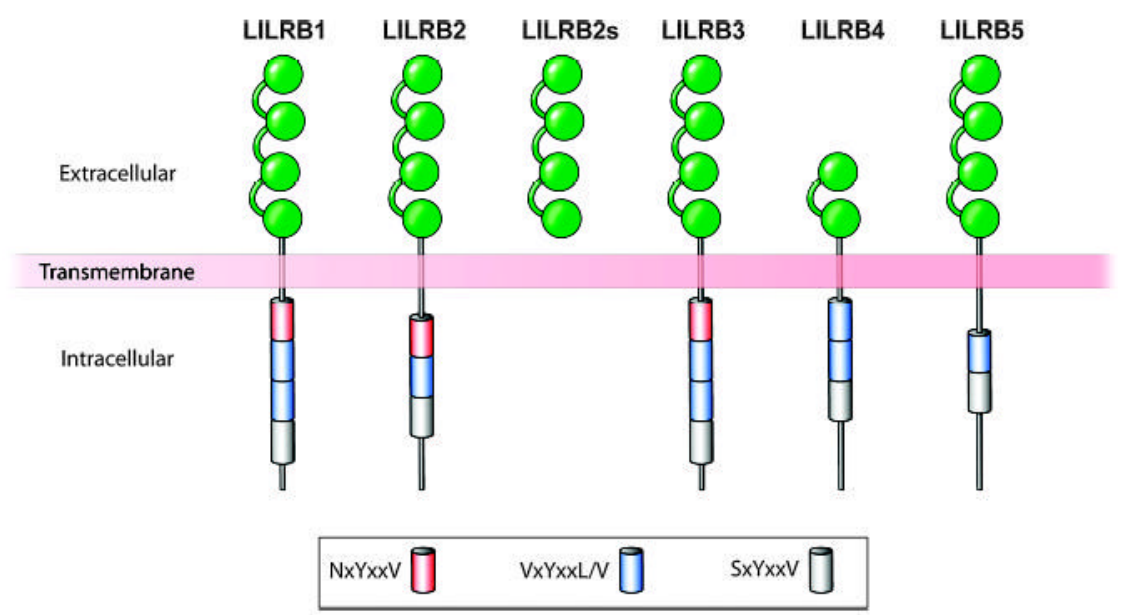

Fig. 5. An illustration of the inhibitory members of the LILR family The inhibiting LILRs lack the arginine residue that is characteristic of the activating members of this family and have varying configurations of the extracellular portions of the molecules. LILRB1 (ILT2), LILRB2 (ILT4), and LILRB3 through B5 have long cytoplasmic tails with two to four immunoreceptor tyrosine-based inhibitory motif domains of varying sequences. Leukocytes express one or more of the LILRB receptors. Modified from Brown et al.(66). 


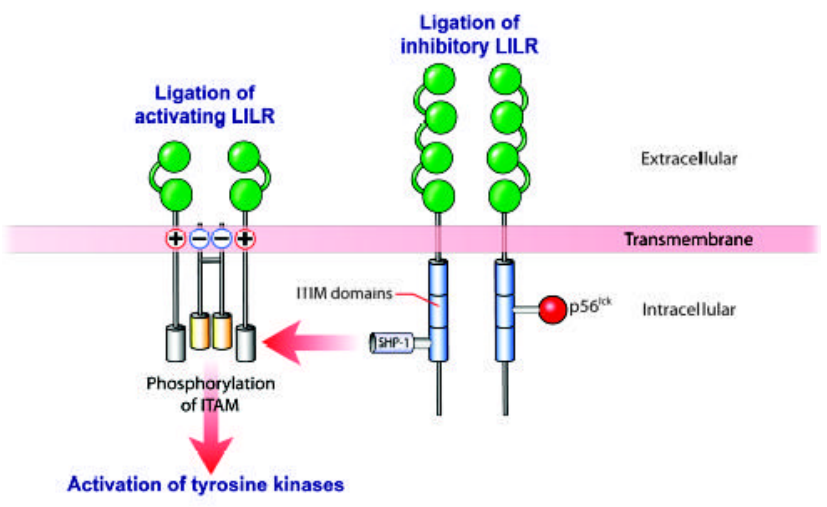

Fig. 6. Inhibitory LILR cancel activating signals

Ligation of inhibitory LILR results in phosphorylation of their ITIM domains and recruitment of $\mathrm{p} 56^{\mathrm{lck}}$ and SHP-1. This causes dephosphorylation of the ITAMS that would, in the absence of the inhibitory signals, drive cell activation. HLA-G interacts with inhibitory LILR. Modified from Brown et al.(66). ITAMS, immunoreceptor tyrosine-based activating motifs; ITIM, immunoreceptor tyrosine-based inhibitory motif; SHP-1, SH2-containing protein-tyrosinephosphatase-1. 


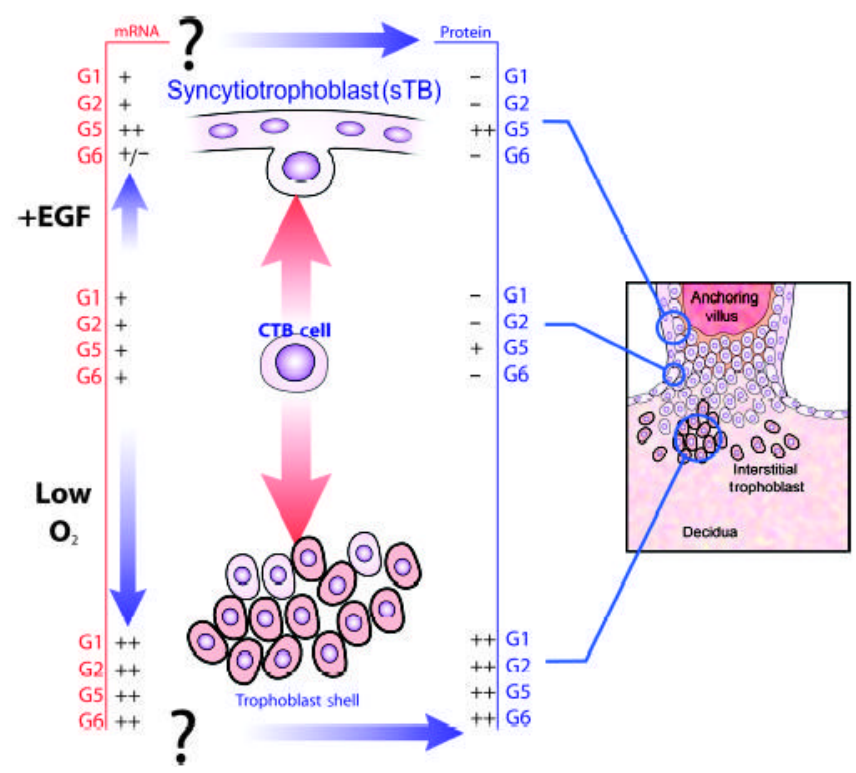

Fig. 7. Multiple conditions regulate expression of HLA-G isoforms in trophoblast cells The vCTB cell (center) may proliferate and drive toward the maternal decidua (lower portion of figure). Acquisition of higher levels of isoform-specific HLA-G mRNAs occurs as a consequence of low oxygen levels in the decidua. Forces driving protein expression in these cells are not known. Alternatively, vCTB cells may differentiate into sTB (upper portion of figure). Epidermal growth factor appears to increase HLA-G5 and reduce HLA-G6 mRNA in these cells and to promote exclusive expression of HLA-G5 in the differentiated syncytium. 


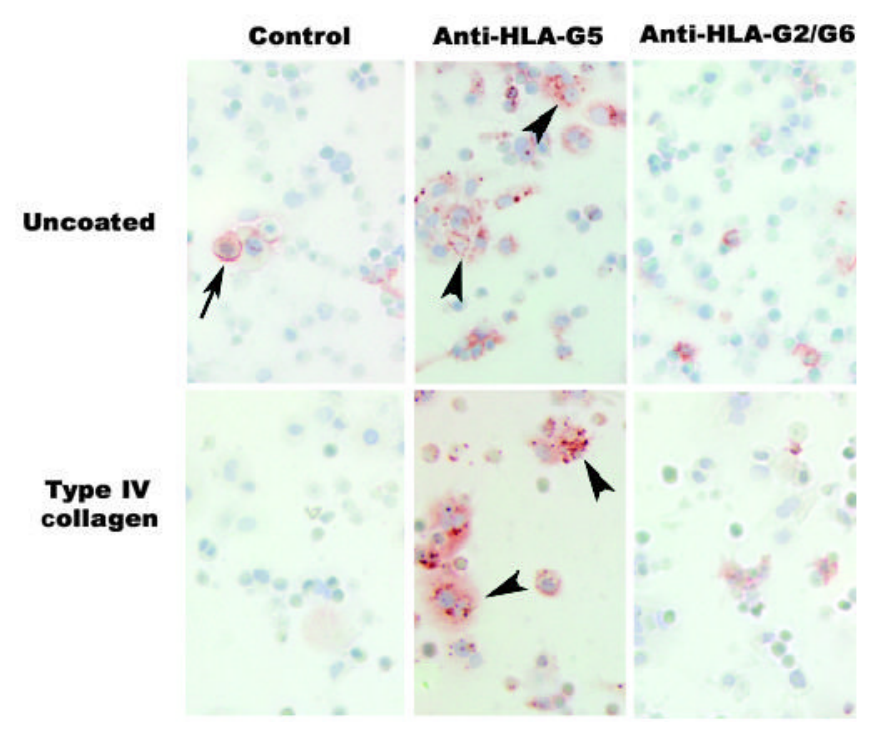

Fig. 8. Substrate influences expression of HLA-G5 but not HLA-G6 in vCTB cells vCTB cells grown on uncoated plates for $48 \mathrm{~h}$ show granular HLA-G5 in cell cytoplasm (arrowheads), whereas cells grown on plates coated with type IV collagen have large intracellular inclusions of HLA-G5 (arrowheads). HLA-G2/G6 appeared to be unchanged. FcR binding of the isotype-specific control monoclonal antibody was more frequent in vCTB cells grown on uncoated plates (arrow) than on coated plates. Original magnifications $\times 200$. 


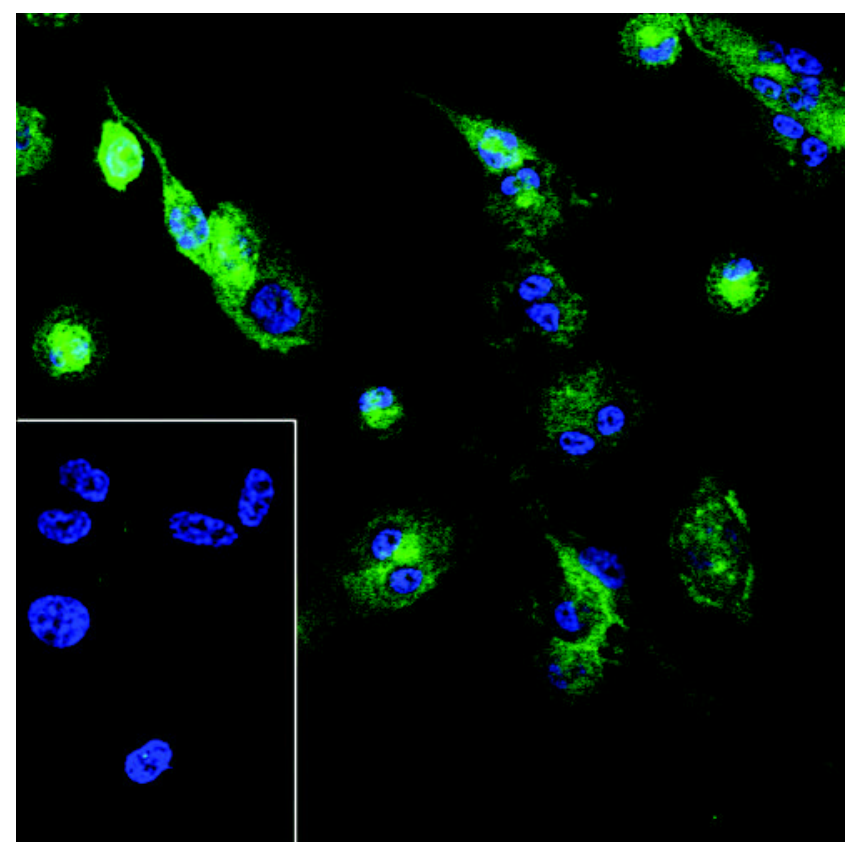

Fig. 9. Immunofluorescent staining for syntaxin-4, an intracellular transport protein (SNARE), in mononuclear phagocyte-like THP-1 cells

This SNARE is present in the monocyte cell line THP-1 following differentiation with phorbol myristate acetate for $24 \mathrm{~h}$ and activation with IFN $\gamma$ for an additional $24 \mathrm{~h}$. Preliminary experiments suggest that proteins such as syntaxin- 4 are altered in these cells following treatment with rHLA-G5, which could affect secretion of cytokines. Blue signal, DAPI nuclear stain; green signal, anti-syntaxin-4. The insert shows negative results with the isotype control for anti-syntaxin-4. Original magnifications $\times 250$. 
Table 1

Mechanisms of tolerance at the maternal-fetal interface in species with hemochorial placentation

\begin{tabular}{|c|c|c|c|}
\hline Type of protection & Species & Genes/proteins & Reference \\
\hline \multirow[t]{2}{*}{ Antibody/complement lysis } & Human & CD46, DAF, CD59 & Kemper et al. (71) \\
\hline & Mice & Crry & $\mathrm{Xu}$ et al. (18) \\
\hline Low lymphocyte proliferation & Human & Progesterone & $\begin{array}{l}\text { Ragusa et al. (72) and Hunt et al. } \\
\text { (73) }\end{array}$ \\
\hline Suppressor lymphocyte generation & Human & IL- 10 , TGF- $\beta 1$ & $\begin{array}{l}\text { Lysiak et al. (15), McIntire et al. } \\
\text { (16), and Wegmann et al. (17) }\end{array}$ \\
\hline Regulation of HLA genes & Human & $H L A-G, H L A-E$ & $\begin{array}{l}\text { Hunt et al. }(5,6) \text {, Hunt }(7), \text { Hunt and } \\
\text { Orr }(32) \text {, and Le Bouteiller and } \\
\text { Mallet (33) }\end{array}$ \\
\hline Regulation of MHC class I genes & Primates & Мати-AG, Paan-AG & $\begin{array}{l}\text { Langat et al. }(22,23) \text { and Slukvin et } \\
\text { al. }(74)\end{array}$ \\
\hline \multirow[t]{2}{*}{ Prevent T-cell killing } & Mice & FasL & Hunt et al. (27) \\
\hline & Human & FasL & Kauma et al. (28) \\
\hline Prevent T-cell killing & Mice & Indoleamine 2,3-dioxygenase & Baban et al. (75) \\
\hline Prevent T-cell killing & Human & $\begin{array}{l}\text { TNF-related apoptosis-inducing } \\
\text { ligand (decoy receptors) }\end{array}$ & Phillips et al. (26) \\
\hline Prevent T-cell killing & Human & TNF (soluble receptors) & Lien et al. (76) \\
\hline Prevent T-cell killing & Human & B7H1 & Petroff et al. (77) \\
\hline
\end{tabular}

